\title{
Drift Modified Longitudinal Electrokinetic Mode in Colloids Laden Semiconductor Quantum Plasmas
}

\author{
Sandhya Chaudhary, Nilesh Nimje, Nishchhal Yadav, and S. Ghosh \\ School of Studies in Physics, Vikram University, Ujjain, Madhya Pradesh 456010, India \\ Correspondence should be addressed to Sandhya Chaudhary; chaudhary.sandhya6@gmail.com
}

Received 13 May 2014; Accepted 28 August 2014; Published 15 September 2014

Academic Editor: Gautam Vemuri

Copyright (C) 2014 Sandhya Chaudhary et al. This is an open access article distributed under the Creative Commons Attribution License, which permits unrestricted use, distribution, and reproduction in any medium, provided the original work is properly cited.

Dispersion and absorption characteristics of electrokinetic wave in unmagnetised extrinsic semiconductor with streaming carriers are analytically investigated. By using quantum hydrodynamic model, a linear dispersion relation is derived for longitudinal electrokinetic wave in colloids laden semiconductor plasma under slow electrokinetic mode regime. Results indicate that quantum effect through Bohm potential significantly modifies the dispersion and absorption characteristics of electrokinetic wave spectrum. The outcome is hoped to add substantially to the present knowledge of wave spectrum of longitudinal electrokinetic wave in colloids laden quantum semiconductor plasma subjected to a dc electric field along the direction of wave propagation.

\section{Introduction}

Due to its potential applications in nanosized electronic devices $[1,2]$, quantum plasma have lately become main thrust area of plasma scientists. Several researchers [3-10] have reported quantum effect in high density and extremely low temperature plasma media, since in these environments the de-Broglie wavelength of the plasma particles become comparable to the Debye length or other scale lengths of the plasma and consequently quantum mechanical effects become important parameter in deciding the behaviour of charged particles.

At extremely low temperatures, due to high value of dielectric response function, the concept of plasma state of semiconductor becomes valid and sensible. The plasmas in solids do not act exactly like free carrier gaseous plasma under external force due to interaction of atoms of lattices and collision effects arise from defects and vibrations of crystalline lattice [11].

Meanwhile, a growing interest in what is known as dusty plasma has appeared $[12,13]$. It may very safely be assumed that the presence of implanted charged colloids can strongly influence the host semiconductor medium and therefore the various properties well studied in dusty plasma can be revisited in this newly thought complex plasma medium in semiconductors. In this way, the presence of colloids in addition to mobile charge carriers already present in semiconductors resembles the dusty gaseous plasma system, as revealed from the references [14-17].

Inspired by present status of both quantum plasma and complex plasma medium in semiconductors, in the current paper, we report, the analytical study of the wave spectrum of electrokinetic waves in colloids laden highly doped semiconductor at liquid nitrogen temperature and the effect of streaming carriers due to presence of strong dc electric field. We firmly believe that the interaction of charged colloids with streaming carriers of host materials might become responsible for the modifications of excited modes and this basic negative-energy carrying mode shall be a very well suited probe for the study of colloids laden semiconductor quantum plasma. Thus, this probe may become very useful to study wave phenomena.

\section{Theoretical Formulation}

We have considered the uniform plasma present in group IV-semiconductor. Let the implantation of metal ions in 
the semiconductor result into the formation of colloids. We have placed the sample in static electric field $\vec{E}_{0}$, applied along negative $z$-direction so that electrons are drifted towards positive $z$-and holes towards negative $z$-directions. The implanted colloids tend to acquire a net negative charge due to higher mobility of electrons compared to that of holes. These colloids are assumed to be remain stationary in the background by considering them to be massive enough to respond to the considered perturbations and regarded as just another component of the plasma medium. We have assumed that all the colloids can be treated as negatively charged point masses. Hence, now the plasma present in group IV-semiconductor that contains electrons, holes, and negatively charged colloids, may be treated with multicomponent plasma model.

To describe such multicomponent plasma system and to include quantum correction term, we have used the quantum hydrodynamic model of plasmas given by Manfredi [18]. The required basic continuity and momentum transfer equations are given by

$$
\begin{gathered}
m_{s} n_{0 s}\left[\partial_{t} v_{z 1 s}+\vartheta_{0 s} \nabla \vartheta_{z 1 s}+\nu_{s} \vartheta_{z 1 s}\right] \\
=z_{s} q_{s} n_{0 s} E_{z 1}-\nabla P_{s}+B_{P} \\
\partial_{t} n_{1 s}+n_{0 s} \nabla \vartheta_{1 s}+\vartheta_{0 s} \nabla n_{1 s}=0 .
\end{gathered}
$$

The quantum statistics is included in the model through the equation of state given below which takes into account Fermionic character of the carriers:

$$
P_{s}=\frac{m_{s} \vartheta_{F s}^{2} n^{3}}{3 n_{0 s}^{2}}
$$

This contribution may be interpreted as quantum pressure term with $\vartheta_{F s}^{2}=2 k_{B} T_{F} / m_{s}$ being the Fermi speed, $k_{B}$ is the Boltzmann constant, and $T_{F}$ is Fermi temperature of carriers. Equation (1) actually represents the equation of motion in quantum hydrodynamic regime with quantum Bohm potential [19] $B_{p}$ given as

$$
B_{p}=\left(\frac{\hbar^{2}}{4 m_{s}}\right)\left\{\nabla\left(\nabla^{2} n_{1 s}\right)\right\} .
$$

The quantum hydrodynamic model is a generalization of classical fluid model of plasmas where the transport equations are expressed in terms of conservation laws for particle, momentum, and energy. The QHD model is a reduced model that allows straight forward investigation of the collective dynamics rather than to deal with complexity of other models. Using this QHD model and following the procedure adopted by Steele and Vural [20], we could obtain the following general dispersion relation for longitudinal electrokinetic mode:

$$
\begin{aligned}
& 1+\frac{\omega_{p e}^{2}}{\left\{\left(\omega-k \vartheta_{0 e}\right)^{2}-i v_{e}\left(\omega-k \vartheta_{0 e}\right)-k^{2} \vartheta_{F e}^{2}\left(1+\Gamma_{e}\right)\right\}} \\
& +\frac{\omega_{p h}^{2}}{\left\{\left(\omega+k \vartheta_{0 h}\right)^{2}-i v_{h}\left(\omega+k \vartheta_{0 h}\right)-k^{2} \vartheta_{F h}^{2}\left(1+\Gamma_{h}\right)\right\}} \\
& +\frac{\omega_{p d}^{2}}{\omega^{2}}=0,
\end{aligned}
$$

where $\omega_{p s}^{2}\left(=\left(z_{s} e\right)^{2} n_{0 s} / \varepsilon m_{s}\right)$ in which $n_{0 s}$ are unperturbed number density of the plasma species $s$ (in which $s=e$ for electrons, $s=h$ for holes, and $s=d$ for dust), $z_{s}$ is the charge states of carriers, $z_{d}\left(=q_{d} / e\right)$ is the ratio of negative charges $q_{d}$ resided over the colloidal grains to the charge $e$ on electrons, $\vartheta_{0 s}\left(=e E_{0} / m_{s} v_{s}\right)$ is the drift velocity of carriers, and $\Gamma_{s}\left(=\hbar^{2} k^{2} / 8 m_{s} k_{B} T_{F}\right)$ in which $\hbar$ is the Plank's constant divided by $2 \pi$.

It is not easy to achieve fast electrokinetic mode in presence of drifting carriers in the medium; hence, we will study this dispersion relation under slow electrokinetic mode situation only. If the phase velocity of the wave is less than the average velocities of electrons and holes both, the mode may be termed as slow electrokinetic mode. Therefore, for slow electrokinetic mode, under collision dominated or low frequency regime $\left[\nu_{e, h} \gg\left(\omega \mp k \vartheta_{0 e, h}\right)\right]$, the dispersion relation reduces to following polynomial in $\omega$ :

$$
A_{4}\left(\omega^{4}\right)+A_{3}\left(\omega^{3}\right)+A_{2}\left(\omega^{2}\right)+A_{1}(\omega)+A_{0}=0
$$

where

$$
\begin{gathered}
\begin{array}{c}
A_{4}=1 \\
\begin{aligned}
A_{3}=- & i\left[\omega_{\mathrm{Re}}\left\{k^{2} \lambda_{F e}^{2}\left(1+\Gamma_{e}\right)-1\right\}\right. \\
& \left.+\omega_{R h}\left\{k^{2} \lambda_{F h}^{2}\left(1+\Gamma_{h}\right)-1\right\}-i\left\{k\left(\vartheta_{0 e}-\vartheta_{0 h}\right)\right\}\right]
\end{aligned} \\
\begin{array}{r}
A_{2}=-\left[k ^ { 2 } \left\{k^{2} \lambda_{F e}^{2}\left(1+\Gamma_{e}\right) \lambda_{F h}^{2}\left(1+\Gamma_{h}\right)-\lambda_{F e}^{2}\left(1+\Gamma_{e}\right)\right.\right. \\
\left.-\lambda_{F h}^{2}\left(1+\Gamma_{h}\right)\right\} \omega_{\mathrm{Re}} \omega_{R h}-\omega_{P d}^{2}+k^{2} \vartheta_{0 e} \vartheta_{0 h}
\end{array} \\
+i\left\{k^{3}\left(\omega_{\mathrm{Re}} \vartheta_{0 e} \lambda_{F e}^{2}\left(1+\Gamma_{e}\right)-\omega_{R h} \vartheta_{0 h} \lambda_{F h}^{2}\left(1+\Gamma_{h}\right)\right)\right. \\
\left.\left.+k\left(\vartheta_{0 e} \omega_{\mathrm{Re}}-\vartheta_{0 h} \omega_{R h}\right)\right\}\right] \\
A_{1}=-i\left[k^{2} \omega_{p d}^{2}\left\{\omega_{\mathrm{Re}} \lambda_{F e}^{2}\left(1+\Gamma_{e}\right)-\omega_{R h} \lambda_{F h}^{2}\left(1+\Gamma_{h}\right)\right\}\right. \\
\left.-i k \omega_{p d}^{2}\left(\vartheta_{0 e}-\vartheta_{0 h}\right)\right]
\end{array}
\end{gathered}
$$




$$
\begin{aligned}
A_{0}=-k^{2}[ & \left\{k^{2} \omega_{P d}^{2} \omega_{\mathrm{Re}} \omega_{R h} \lambda_{F e}^{2}\left(1+\Gamma_{e}\right) \lambda_{F h}^{2}\left(1+\Gamma_{h}\right)\right. \\
& \left.+\omega_{p d}^{2} \vartheta_{0 e} \vartheta_{0 h}\right\} \\
+ & i k\left\{\vartheta_{0 e} \omega_{p d}^{2} \omega_{\mathrm{Re}} \lambda_{F e}^{2}\left(1+\Gamma_{e}\right)\right. \\
& \left.\left.-\vartheta_{0 h} \omega_{p d}^{2} \omega_{R h} \lambda_{F h}^{2}\left(1+\Gamma_{h}\right)\right\}\right]
\end{aligned}
$$

in which $\omega_{\mathrm{Re}, h}\left(=\omega_{p e, h}^{2} / \nu_{e, h}\right)$ are the dielectric relaxation frequencies and $\lambda_{F e, h}\left(=\vartheta_{F e, h} / \omega_{p e, h}\right)$ is the Fermi screening scale length of electrons and holes, respectively.

Now, it may be inferred from (6) that the quantum effect introduced through quantum terms $\Gamma_{s}$ effectively modifies the wave spectra of all the four possible modes. Equation (6) being of fourth degree in complex wave frequency $(\omega=$ $\beta+i \alpha$ ) with complex coefficient is not easy to solve analytically and so we solved it numerically using La Guerre method of finding roots of polynomial.

\section{Results and Discussion}

The form of perturbations was considered as $\exp [i(\omega t-k z)]$ and so the mode may be growing in time when imaginary part of the wave angular frequency becomes negative for the real value of $k$; that is, $\alpha<0$ and decaying when $\alpha>0$. In order to study the behavior of propagating modes, where the electrons and holes are drifting in opposite direction with drift velocities $+\vartheta_{0 e}$ and $-\vartheta_{0 h}$, respectively, we use $\omega=\beta+i \alpha$, in (6), and solve it numerically, where $\beta$ is the real frequency of wave mode and $\alpha$ is the growth rate.

We have applied the above analysis to the case of group IV-semiconductor Ge with the following constants: $m_{e}=$ $0.0815 m_{0}, m_{0}$ being the free electron mass, $m_{h}=4 m_{e}, m_{d}=$ $10^{-27} \mathrm{~kg}, \varepsilon_{L}=15.8, n_{0 e}=10^{22} \mathrm{~m}^{-3}, n_{0 h}=5 \times 10^{22} \mathrm{~m}^{-3}, n_{0 d}=$ $10^{16} \mathrm{~m}^{-3}, v_{e}=3.463 \times 10^{11} \mathrm{sec}^{-1}, v_{h}=1.194 \times 10^{11} \mathrm{sec}^{-1}$, and $v_{d}=3.422 \times 10^{8} \mathrm{sec}^{-1}$ at $77 \mathrm{k}$.

Figures 1 to 4 display the dispersion and absorption characteristics of all the four possible modes including and excluding quantum effect $(\mathrm{QE})$ with carrier drift in term of dc electric field $E_{0}$.

Figures 1 and 2 show the dispersion characteristics of the propagating wave (i.e., I-, II-, III-, and IV-modes) in ionimplanted quantum semiconductor plasma medium. Figure 1 shows the variation of $\beta$ with $E_{0}$ of I- and II-modes in presence and absence of QE. It is clear from Figure 1 that both the modes (I and II) are propagating in opposite direction to each other, that is, I-mode propagating in the direction of wave propagation ( $-z$ axis) whereas II-mode propagating opposite direction of wave propagation with equal phase speed. Magnitude of phase constant of I- and II-modes excluding quantum effect increase slowly with increment in carrier drift but including $\mathrm{QE}$ phase constant is nearly nonvariant up to $E_{0}=4.3 \times 10^{5} \mathrm{Vm}^{-1}$. Further, if we increase the carrier drift, the phase speed of both modes decreases with it. Phase constant of I- and II-modes including $\mathrm{QE}$ decreases more rapidly as compared to that obtained

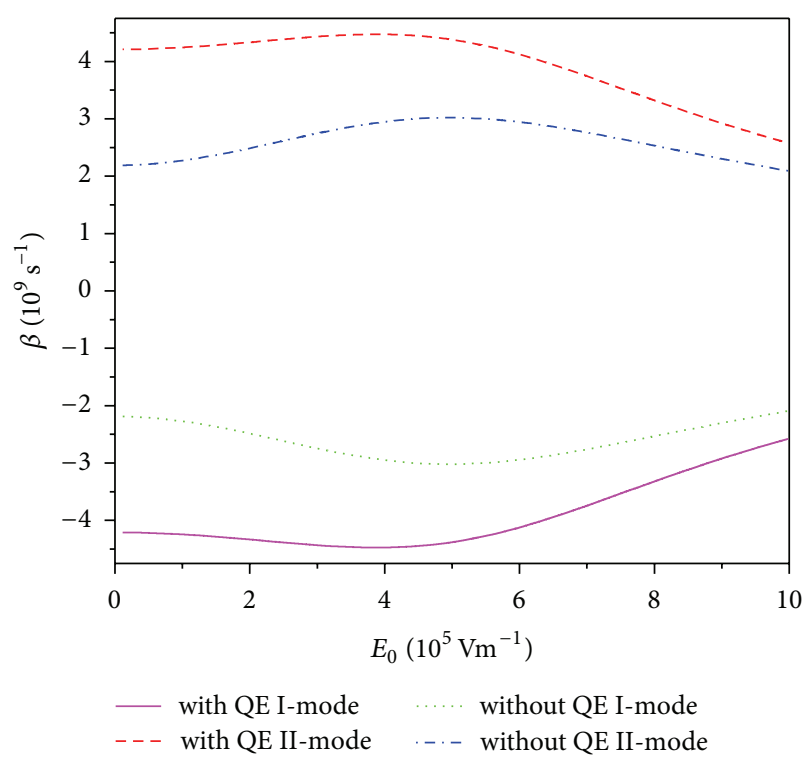

FIgURE 1: The real frequency $\beta$ versus the carrier drift in term of electric field $E_{0}$ for I- and II-modes in presence and absence of QE where $k=5 \times 10^{7} \mathrm{~m}^{-1}$.

excluding QE. Here, it is confirmed that the phase speed of both modes modified in presence of QE.

Figure 2 infers that the phase speed of both (III and IV) modes including and excluding QE increases with increasing carrier drift in terms of $E_{0}$. It is clearly seen from this figure that IV-mode is copropagating mode and III-mode is counter-propagating mode. Inclusion of QE enhances the phase speed of both the modes. By increasing the strength of electric field, the phase velocity of III-mode increases in presence and absence of QE. The phase speed of III-mode is slightly higher than the IV-mode in presence as well as absence of QE. Hence, it is confirmed that the quantum effect in multicomponent semiconductor plasma is responsible for modification of phase speed of both the modes (III and IV).

Figures 3 and 4 show the absorption characteristics of all four possible modes of slow electrokinetic mode in presence and absence of $\mathrm{QE}$ with carrier drift in terms of $E_{0}$. From Figure 3, it may easily be illustrated that the characteristics of both the modes (I and II) are exactly opposite; the I-mode is growing (amplifying) with increment in carrier drift whereas the II-mode is decaying with it. Inclusion of QE enhances the phase speed of both the modes as well as enhances the growth rate of I-mode and attenuation coefficient of II-mode.

In Figure 4, the characteristics of III- and IV-modes are found to be again of opposite nature to each other; that is, III-mode is found to be decaying in nature whereas IV-mode is growing in nature in presence and absence of QE. It is also clear that the decay and growth rates of III- and IV-modes slowly decrease with increment in carrier drift. The decay rate of III-mode is higher in presence of QE whereas QE decreases the growth rate of IV-mode. 


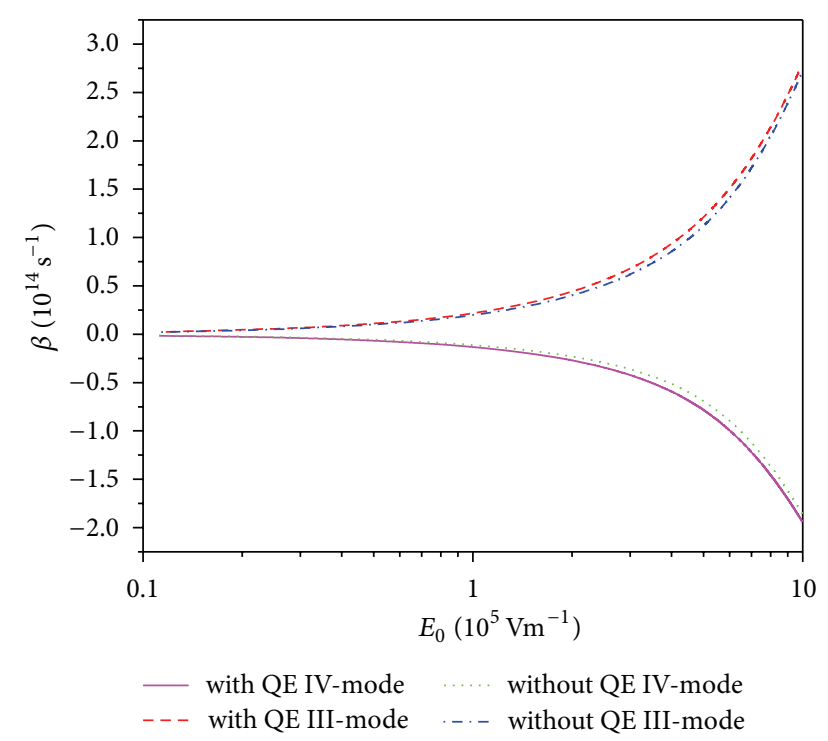

Figure 2: The real frequency $\beta$ versus the carrier drift in term of electric field $E_{0}$ for III- and IV-modes in presence and absence of QE where $k=5 \times 10^{7} \mathrm{~m}^{-1}$.

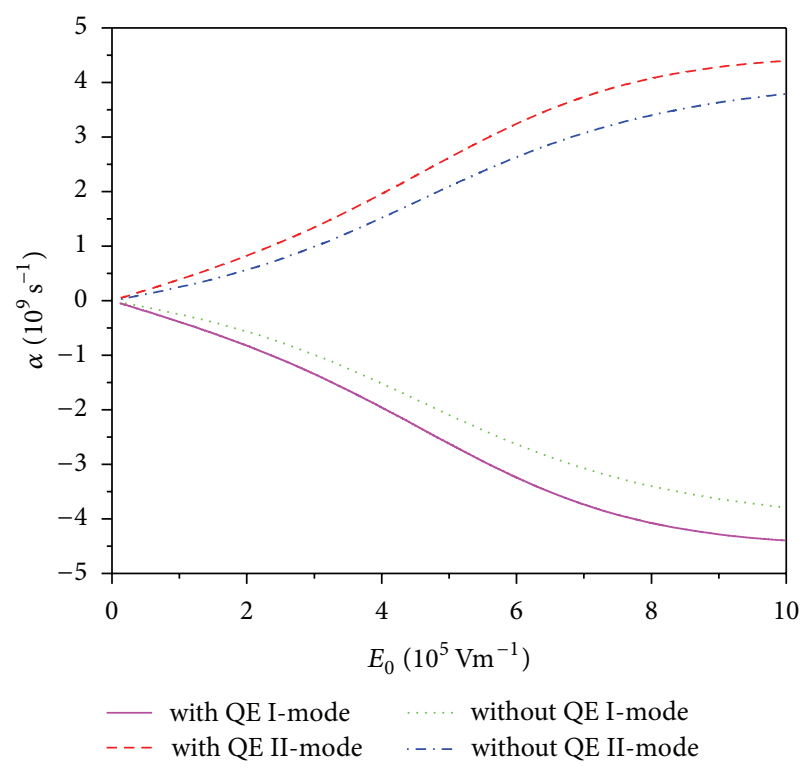

FIgURE 3: The gain coefficients $\alpha$ versus the carrier drift in terms of electric field $E_{0}$ for I- and II-modes in presence and absence of QE where $k=5 \times 10^{7} \mathrm{~m}^{-1}$.

\section{Conclusions}

In this investigation, we have studied drift modified longitudinal electrokinetic mode in colloids laden semiconductor quantum plasma using QHD model under the slow electrokinetic mode limit. It is found that quantum effect through Bohm potential and the effect of streaming carriers due to presence of strong dc electric field significantly modify the propagation characteristics of all the modes. We also found that the quantum correction term substantially alters the dispersion and absorption characteristics of electrokinetic

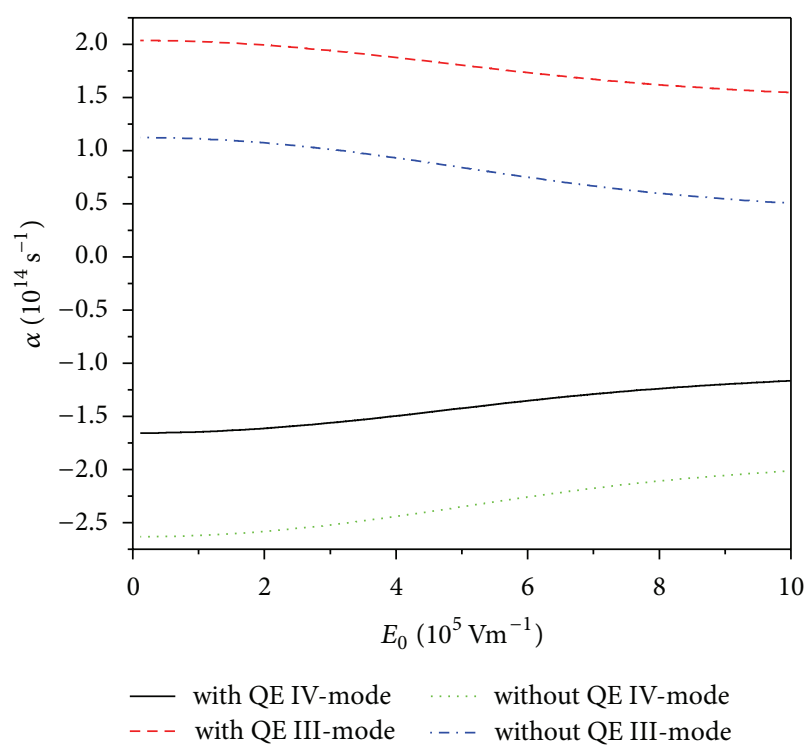

FIGURE 4: The gain coefficients $\alpha$ versus the carrier drift in terms of electric field $E_{0}$ for III- and IV-modes in presence and absence of quantum effect where $k=5 \times 10^{7} \mathrm{~m}^{-1}$.

wave in colloid laden semiconductor plasma when sample is highly doped. The weakness of QHD model, of-course, results in its inability to take into account kinetic effects like landau damping, driven by resonant wave-particle interactions.

\section{Conflict of Interests}

The authors declare that there is no conflict of interests regarding the publication of this paper.

\section{Acknowledgment}

The financial assistance from the Madhya Pradesh Council of Science and Technology, Bhopal, India, under a research project is gratefully acknowledged.

\section{References}

[1] Y.-D. Jung, "Quantum-mechanical effects on electron-electron scattering in dense high-temperature plasmas," Physics of Plasmas, vol. 8, no. 8, pp. 3842-3844, 2001.

[2] K.-Z. Zhang and J.-K. Xue, "Streaming instability in bounded three-component quantum plasmas," Physics of Plasmas, vol. 17, Article ID 032113, 2010.

[3] F. Haas, G. Manfredi, and M. Feix, "Multistream model for quantum plasmas," Physical Review E-Statistical Physics, Plasmas, Fluids, and Related Interdisciplinary Topics, vol. 62, no. 2 B, pp. 2763-2772, 2000.

[4] S. Ali and P. K. Shukla, "Streaming instability in quantum dusty plasmas," The European Physical Journal D, vol. 41, no. 2, pp. 319324, 2007.

[5] N. Shukla, P. K. Shukla, G. Brodin, and L. Stenflo, "Ion streaming instability in a quantum dusty magnetoplasma," Physics of Plasmas, vol. 15, no. 4, Article ID 044503, 2008. 
[6] M. Salimullah, M. Jamil, H. A. Shah, and G. Murtaza, "Jeans instability in a quantum dusty magnetoplasma," Physics of Plasmas, vol. 16, no. 1, Article ID 014502, 2009.

[7] M. Salimullah, M. Jamil, I. Zeba, C. Uzma, and H. A. Shah, "Drift wave instability in a nonuniform quantum dusty magnetoplasma," Physics of Plasmas, vol. 16, no. 3, Article ID 034503 , 2009.

[8] A. Hussain, I. Zeba, M. Salimullah, G. Murtaza, and M. Jamil, "Modified screening potential in a high density inhomogeneous quantum dusty magnetoplasma," Physics of Plasmas, vol. 17, no. 5, Article ID 054504, 2010.

[9] I. Zeba, C. Uzma, M. Jamil, M. Salimullah, and P. K. Shukla, "Colloidal crystal formation in a semiconductor quantum plasma," Physics of Plasmas, vol. 17, no. 3, Article ID 032105, 2010.

[10] M. Jamil, M. Shahid, W. Ali, M. Salimullah, H. A. Shah, and G. Murtaza, "The parametric decay of dust ion acoustic waves in non-uniform quantum dusty magnetoplasmas," Physics of Plasmas, vol. 18, Article ID 063705, 2011.

[11] G. Brodin and M. Marklund, "Spin magnetohydrodynamics," New Journal of Physics, vol. 9, article 277, 2007.

[12] M. Bacha, M. Tribeche, and P. K. Shukla, "Dustion-acoustic solitary waves in a dusty plasma with nonextensive electrons," Physical Review E-Statistical, Nonlinear, and Soft Matter Physics, vol. 85, no. 5, Article ID 056413, 2012.

[13] I. Tasnim, M. M. Masud, and A. A. Mamun, "Effects of nonthermal ions of distinct temperatures on dust acoustic shock waves in a dusty plasma," Astrophysics and Space Science, vol. 343, no. 2, pp. 647-652, 2013.

[14] M. Salimullah, P. K. Shukla, S. K. Ghosh, H. Nitta, and Y. Hayashi, "Electron-phonon coupling effect on wakefields in piezoelectric semiconductors," Journal of Physics D: Applied Physics, vol. 36, no. 8, pp. 958-960, 2003.

[15] M. Salimullah, A. M. Rizwan, S. K. Ghosh et al., "Long-ranged order formation of colloids of implanted ions in a dc biased piezoelectric semiconductor," Journal of Applied Physics, vol. 97, no. 12, Article ID 124505, 2005.

[16] S. Ghosh and P. Thakur, "Instability of circularly polarized electro-kinetic waves in magnetized ion-implanted semiconductor plasmas," European Physical Journal D, vol. 37, no. 3, pp. 417-422, 2006.

[17] S. Ghosh, P. Thakur, and M. Salimullah, "Dispersion and absorption of longitudinal electro-kinetic waves in ionimplanted semiconductor plasmas," Indian Journal of Pure and Applied Physics, vol. 44, no. 3, pp. 235-242, 2006.

[18] G. Manfredi, "How to model quantum plasma," Fields Institute Communication, vol. 46, p. 263, 2005.

[19] D. Bohm, "A suggested interpretation of the quantum theory in terms of "hidden" variables. I," Physical Review, vol. 85, p. 166, 1952.

[20] M. C. Steele and B. Vural, Wave Interaction in Solid State Plasmas, Mc-Graw Hill, New York, NY, USA, 1969. 

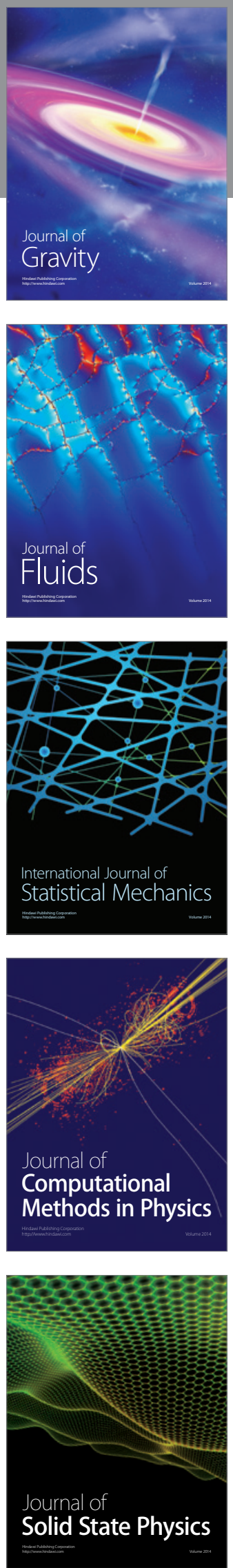

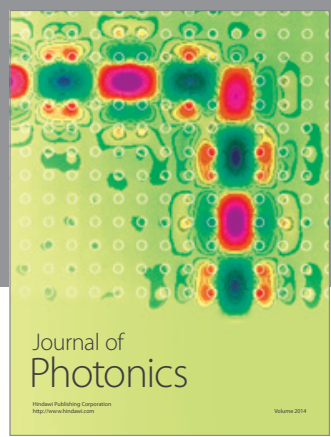

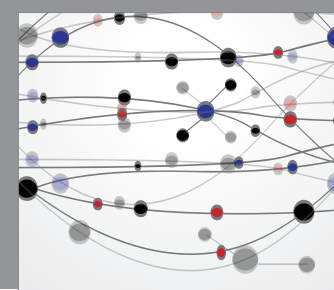

The Scientific World Journal

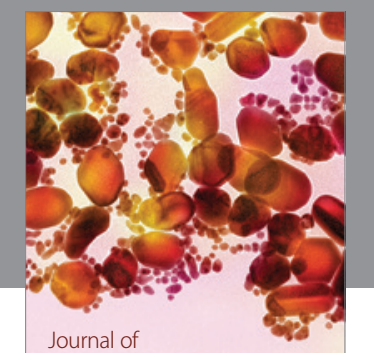

Soft Matter
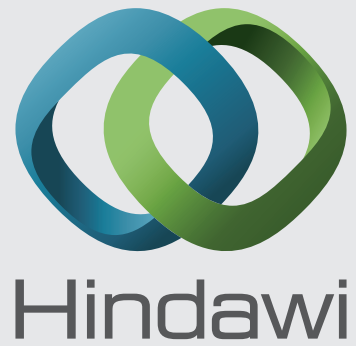

Submit your manuscripts at

http://www.hindawi.com
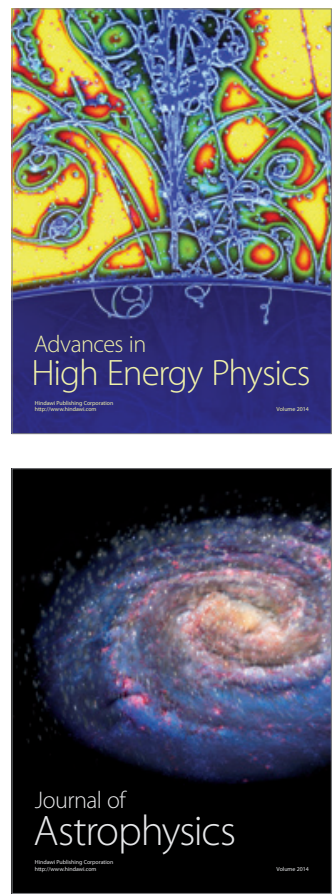
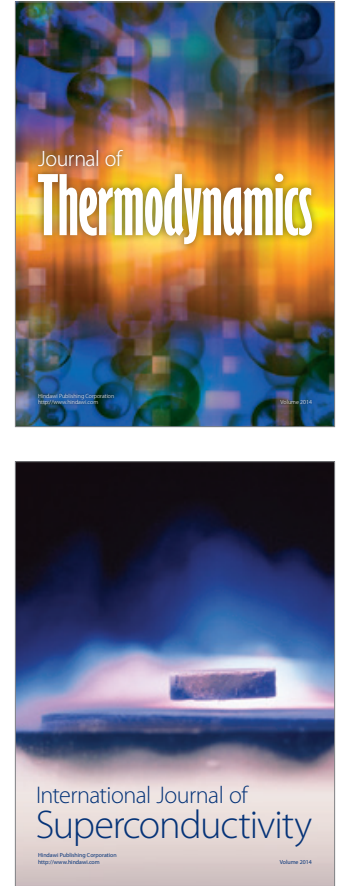
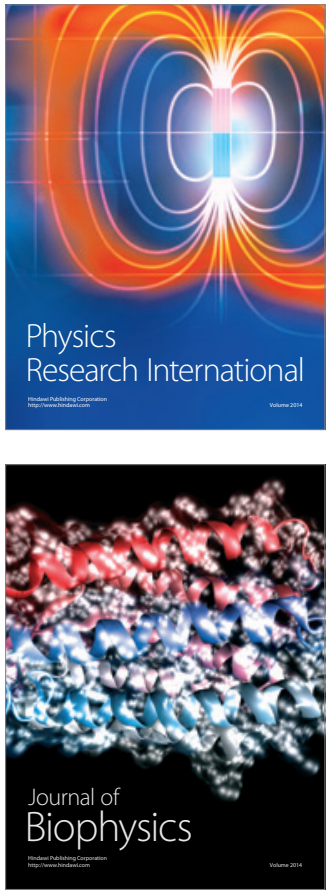
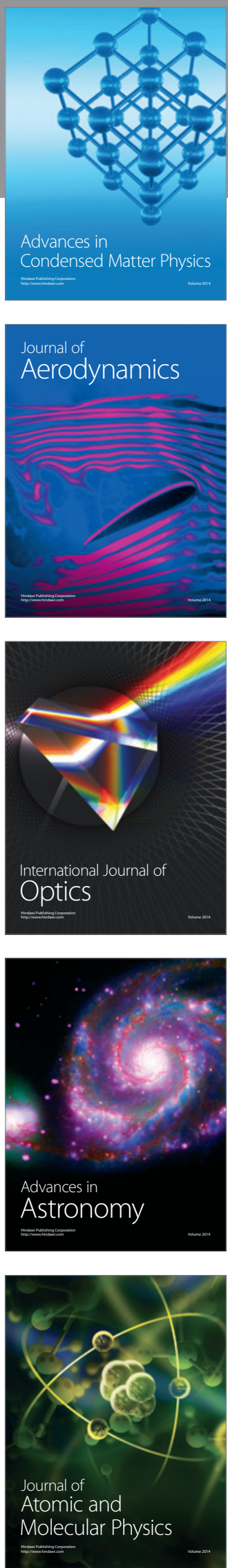